\title{
Quantitative proofs of certain Algebraic Inequalities.
}

\section{By John Dougatl, M.A.}

1. By a quantitative proof of an inequality I mean one which exhibits the difference between the two magnitudes compared in a form which shows at a glance whether the difference is positive or negative. Such a proof not merely establishes the existence of the inequality, but also gives a measure of its amount.

The formula $\quad a^{2}+b^{2}-2 a b=(a-b)^{2}$, as a proof that $a^{2}+b^{2}>2 a b$, is a characteristic example.

Another is the proof of the important theorem that when $p$ is a positive integer, and $x$ any positive number,

$$
\frac{x^{p+1}-1}{p+1}>\frac{x^{p}-1}{p}
$$

which is contained in the obvious identity

$$
\begin{aligned}
& p\left(x^{p+1}-1\right)-(p+1)\left(x^{p}-1\right) \\
= & (x-1)^{2}\left(p x^{\nu-1}+\overline{p-1} x^{p-2}+\ldots+1\right) .
\end{aligned}
$$

Dr Muirhead, in two papers in Vols. XIX. and XXI. of these Proceedings, has made some interesting applications of the method; in the present communication I give a few additional developments suggested by a perusal of these papers.

The inequalities with which the paper deals admit of very simple qualitative proofs depending on the Theory of Equations, and they have been discussed from that point of view by Euler, Fort, and Schlomilch in the manner indicated in next article.

2. Taking $n$ real positive numbers $a_{1}, a_{2}, \ldots, a_{n}$, form the product

$$
\left(x+a_{1} y\right)\left(x+a_{2} y\right) \ldots \ldots\left(x+a_{n} y\right)
$$


and write its expansion in the form

$$
\mathrm{A}_{0} x^{n}+n \mathbf{A}_{1} x^{n-1} y+\frac{n(n-1)}{1.2} \mathbf{A}_{2} x^{n-2} y^{2}+\ldots+n \mathbf{A}_{n-1} x y^{n-1}+\mathbf{A}_{n} y^{n} .
$$

If we differentiate $n-r-1$ times as to $x$, and $r-1$ times as to $y$, then by a fundamental theorem the resulting quadratic function, which to a constant factor is

$$
\mathrm{A}_{r-1} x^{2}+2 \mathrm{~A}_{r} x y+\mathrm{A}_{r+1} y^{2}
$$

has its linear factors real.

Hence $A_{r}{ }^{2}-A_{r-1} A_{r+1}$ is positive. - - - - - (a)

We have therefore

$$
\frac{A_{1}}{A_{0}}>\frac{A_{2}}{A_{1}}>\frac{A_{3}}{A_{2}}>\ldots>\frac{A_{n}}{A_{n-1}} .
$$

In particular, if $s \Varangle r$

$$
\frac{A_{r}}{A_{r-1}}>\frac{A_{t+1}}{A_{r}}
$$

and $A_{r} A_{a}-A_{r-1} A_{s+1}$ is positive.

Also $\frac{A_{r}}{A_{r-1}} \cdot \frac{A_{r-1}}{A_{r-2}} \ldots \frac{A_{r-p+1}}{A_{r-p}}>\frac{A_{s+1}}{A_{s}} \frac{A_{s+2}}{A_{c+1}} \ldots \frac{A_{s+p}}{A_{t+p-1}}$,

that is $\quad \frac{\mathbf{A}_{r}}{\mathbf{A}_{r-p}}>\frac{\mathbf{A}_{\boldsymbol{r}+p}}{\mathbf{A}_{\mathbf{r}}}$

or $A_{r} A_{a}-A_{r-p} A_{t+p}$ is positive

Again from (a) we have $A_{r}{ }^{2 r}>A_{r-1}^{r} A_{r+1}^{r}$, and therefore

$$
\frac{\mathbf{A}_{r}^{r+1}}{\mathbf{A}_{r+1}^{r}}>\frac{\mathbf{A}_{r-1}^{r}}{\mathbf{A}_{r}^{r-1}}>\frac{\mathbf{A}_{r-9}^{r-1}}{\mathbf{A}_{r-1}^{r-2}}>\ldots \ldots>\frac{\mathbf{A}_{1}^{2}}{\mathbf{A}_{2}}>1
$$

and $A_{r}^{r+1}-A_{r+1}^{r}$ is positive.

Generally, if we raise the inequalities

$$
\begin{aligned}
& A_{1}{ }^{2}>A_{0} A_{2} \\
& A_{2}{ }^{2}>A_{1} A_{3} \\
& A_{3}{ }^{2}>A_{9} A_{4}
\end{aligned}
$$


to the positive integral powers $\alpha_{1}, \alpha_{2}, \alpha_{3}, \ldots$ and multiply, we get

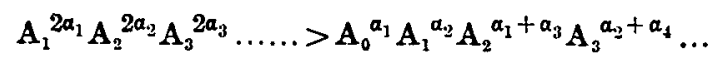

or say, when the common factors are removed

so that

$$
\mathrm{A}_{r}^{\alpha} \mathrm{A}_{s}^{\beta} \ldots \ldots>\mathrm{A}_{p}^{\gamma} \mathrm{A}_{q}^{\delta} \ldots \ldots
$$

$$
A_{r}^{\alpha} A_{z}^{\beta} \ldots \ldots-A_{p}^{\gamma} A_{y}^{\delta} \ldots \ldots \text { is positive. }
$$

Dr Muirhead, in Vol. XXI., proves (a) by expressing $A_{r}{ }^{2}-A_{r-1} A_{r+1}$ as a sum of terms each of which is manifestly positive. The problem proposed here is to do the same thing for the general form (e) and in particular for the special cases $(b),(c),(d)$. Moreover we stipulate that the functions as the sum of which the difference $(e)$ is expressed, besides being patently positive, shall be integral functions of the $a$ 's. This restriction aside, the problem would be extremely simple. In fact, given a number of inequalities between positive quantities, such as

$$
\begin{aligned}
& x_{1}>y_{1} \\
& x_{2}>y . \\
& x_{3}>y_{3}
\end{aligned}
$$

from which we can deduce $x_{1} x_{2} x_{3} .>y_{1} y_{2} y_{3} \ldots$, nothing is easier than to write the difference

$$
x_{1} x_{2} x_{3} \ldots \ldots-y_{1} y_{2} y_{3} \ldots \ldots
$$

in such a form as to show its essentially positive character.

For example, we may write

$$
\begin{aligned}
& x_{1}=y_{1}+\left(x_{1}-y_{1}\right) \\
& x_{2}=y_{2}+\left(x_{2}-y_{2}\right), \text { etc., }
\end{aligned}
$$

and then the extended product of the binomial factors

$$
\left(y_{1}+\overline{x_{1}-y_{1}}\right)\left(y_{2}+\overline{x_{2}-y_{2}}\right) \ldots \ldots
$$

contains $y_{1} y_{2} \ldots$ together with obviously positive terms. 
Or better, we may write

$$
\begin{aligned}
x_{1} x_{2}-y_{1} y_{2} & =x_{2}\left(x_{1}-y_{1}\right)+y_{1}\left(x_{2}-y_{2}\right) \\
x_{1} x_{2} x_{3}-y_{1} y_{2} y_{3} & =\left(x_{1} x_{2}-y_{1} y_{2}\right) x_{3}+y_{1} y_{2}\left(x_{3}-y_{3}\right) \\
& =x_{2} x_{3}\left(x_{1}-y_{1}\right)+y_{1} x_{3}\left(x_{2}-y_{2}\right)+y_{1} y_{2}\left(x_{3}-y_{3}\right)
\end{aligned}
$$

the general result corresponding to which is obvious.

The form (e) before common factors have been removed from the two terms, could be treated in this way and expressed in terms of the differences $(a)$. But the simplified form of $(e)$ and in particular the forms $(b),(c),(d)$ would thus be given as a sum of terms which, while plainly positive, would in general involve powers of the $A$ 's in the denominators,

$$
\text { e.g., } \quad \mathbf{A}_{1} \mathbf{A}_{2}-\mathbf{A}_{0} \mathbf{A}_{3}=\frac{\mathbf{A}_{1}}{\mathbf{A}_{2}}\left(\mathbf{A}_{2}{ }^{2}-\mathbf{A}_{1} \mathbf{A}_{3}\right)+\frac{\mathbf{A}_{3}}{\mathbf{A}_{2}}\left(\mathbf{A}_{1}{ }^{2}-\mathbf{A}_{0} \mathbf{A}_{2}\right) \text {. }
$$

It will be shown, however, that by making use in the way thus suggested of forms of the type $(b)$ in addition to those of the type (a) the difficulty about fractions can be surmounted.

What we do, then, is this:-First, we investigate expressions for $A_{r} A_{s}-A_{r-1} A_{s+1}$ where $s \nless r$, as a sum of evidently positive terms. The method used is different from Muirhead's, but some of the expressions found reduce to those given by him when $r=8$. Second, we express the differences $(c),(d),(e)$ as linear functions of forms of the types $(a)$ and $(b)$, the coeffieients of these functions being products of integral powers of the A's, or sums of such.

3. Given $n$ letters $a, b, c, \ldots$, which in the applications to inequalities we shall suppose to denote positive numbers;

$$
\text { let } \mathbf{P}_{r} \equiv \Sigma a b c \ldots . \text {. to } r \text { factors; }
$$

also let $\mathbf{P}_{0}=1$, and when the integer $r$ is negative or greater than $n$, $\mathrm{P}_{r}=0$.

In $\mathrm{P}_{\tau}$ let $\mathrm{A}=$ sum of the terms containing $a$,

$$
\mathrm{B}=", " \quad, \quad, \quad \text {, etc. }
$$

In. $P_{8}$ let $A^{\prime}=$ sum of the terms not containing $a$,

$$
\mathrm{B}^{\prime}=, \quad, \quad, \quad, \quad, \quad, \quad b \text {, etc. }
$$


Then we have

$$
\begin{aligned}
& \mathrm{A}+\mathrm{B}+\mathrm{C}+\ldots . . .=r \mathrm{P}_{r}, \\
& \mathrm{~A}^{\prime}+\mathrm{B}^{\prime}+\mathrm{C}^{\prime}+\ldots . .=(n-s) \mathrm{P}_{\mathrm{a}}, \\
& \text { and } \frac{\mathrm{A}}{a}+\frac{\mathrm{B}}{b}+\frac{\mathrm{C}}{c}+\ldots . .=(n-r+1) \mathrm{P}_{r-1} \text {; } \\
& a \mathrm{~A}^{\prime}+b \mathrm{~B}^{\prime}+c \mathrm{C}^{\prime}+\ldots . .=(s+1) \mathrm{P}_{s+1}
\end{aligned}
$$

as we see at once by counting the number of times any particular term occurs in the expressions on the left.

Thus $r(n-s) \mathrm{P}_{r} \mathrm{P}_{s}-(n-r+1)(s+1) \mathrm{P}_{r-1} \mathrm{P}_{s+1}$

$$
\begin{aligned}
& =(\mathrm{A}+\mathrm{B}+\ldots)\left(\mathrm{A}^{\prime}+\mathrm{B}^{\prime}+\ldots\right)-\left(\frac{\mathrm{A}}{a}+\frac{\mathrm{B}}{b}+\ldots\right)\left(a \mathrm{~A}^{\prime}+b \mathrm{~B}^{\prime}+\ldots\right) \\
& =\Sigma\left\{\mathrm{AB}^{\prime}(1-b / a)+\mathrm{A}^{\prime} \mathrm{B}(1-a / b)\right\} \\
& =\Sigma(a-b)\left(\frac{\mathrm{A}}{a} \mathrm{~B}^{\prime}-\frac{\mathrm{B}}{b} \mathrm{~A}^{\prime}\right) .-\ldots-\ldots
\end{aligned}
$$

Now we may write

$$
\begin{aligned}
& \mathbf{P}_{r}=a b^{\prime} \mathbf{T}+(a+b) \mathbf{S}+\mathbf{R}, \\
& \mathbf{P}_{\imath}=a b^{\prime} \mathbf{T}^{\prime}+(a+b) \mathbf{S}^{\prime}+\mathbf{R}^{\prime}
\end{aligned}
$$

where $T, S, R, T^{\prime}, S^{\prime}, R^{\prime}$ do not contain $a$ or $b$.

$$
\text { With this notation } \quad \begin{aligned}
& \mathbf{A}=a(b \mathbf{T}+\mathbf{S}), \\
& \mathbf{B}=b(a \mathbf{T}+\mathbf{S}) \\
& \text { and } \quad \mathbf{A}^{\prime}=b \mathbf{S}^{\prime}+\mathbf{R}^{\prime}, \\
& \mathbf{B}^{\prime}=a \mathbf{S}^{\prime}+\mathbf{R}^{\prime} .
\end{aligned}
$$

The typical term on the right of $(1)$ is then

$$
\begin{aligned}
& (a-b)\left\{(b \mathbf{T}+\mathbf{S})\left(a \mathbf{S}^{\prime}+\mathbf{R}^{\prime}\right)-(a \mathrm{~T}+\mathbf{S})\left(b \mathbf{S}^{\prime}+\mathbf{R}^{\prime}\right)\right\} \\
= & (a-b)^{2}\left(\mathbf{S S}^{\prime}-\mathbf{T R}^{\prime}\right\rangle \\
= & (a-b)^{2}\left(\mathbf{P}_{r-1}^{a b} \mathbf{P}_{s-1}^{a b}-\mathbf{P}_{r-2}^{a b} \mathbf{P}_{s}^{a b}\right) .
\end{aligned}
$$

where we use the symbol $P_{t}^{a b}$ to denote $\Sigma_{c d} \ldots$ to $t$ factors taken from the $n-2$ letters left when $a, b$ are excluded from the original $n$.

Hence from (1)

$$
\begin{aligned}
& r(n-s) \mathrm{P}_{r} \mathrm{P}_{s}-(n-r+1)(s+1) \mathrm{P}_{r-1} \mathrm{P}_{a+1} \\
= & \sum(a-b)^{2}\left(\mathrm{P}_{r-1}^{a b} \mathrm{P}_{s-1}^{a b}-\mathrm{P}_{r-2}^{a b} \mathrm{P}_{s}^{a b}\right) . \quad-
\end{aligned}
$$

On this formula the whole of the succeeding work is based. 
4. If the cofactor of $(a-b)^{2}$ in (2) be fully expanded, the numerical coefficient of every term will be positive, provided 8 is not less than $r$, as we shall always suppose to be the case.

For consider $\mathrm{P}_{r} \mathrm{P}_{s}-\mathrm{P}_{r-1} \mathrm{P}_{s+1}$, involving $t$ letters $a_{1}, a_{2}, \ldots, a_{t}$.

The product $P_{r} P_{a}$, when the number of letters is sufficient, involves terms of the types

$$
\begin{aligned}
& a_{1} a_{2} \ldots \ldots, a_{r} . a_{r+1} \ldots a_{r+*}, \text { coefficient }=(r+s) ! /(r ! s !) \\
& a_{1}{ }^{2} a_{2} \ldots \ldots a_{r}, a_{r+1} \ldots a_{r+s-1}, \text { coefficient }=(r+s-2) ! /(r-1) !(s-1) ! \\
& a_{1}{ }^{2} a_{2}^{2} a_{3} \ldots a_{r} . a_{r+1} \ldots a_{r+s-2}, \text { coefficient }=(r+s-4) ! /(r-2) !(s-2) ! \\
& a_{1}^{2} a_{2}^{2} \ldots \ldots a_{r}^{2} \cdot a_{r+1} \ldots a_{s} \quad, \text { coefficient }=1 .
\end{aligned}
$$

When $r+s>t$, some of the earlier terms will not occur, but those terms which do occur have the coefficient stated.

$\mathbf{P}_{r-1} \mathbf{P}_{a+1}$ will involve terms of the same types, except the last, and the coefficients will be

$$
(r+s) ! /(r-1) !(s+1) !,(r+s-2) ! /(r-2) ! s !, \text { etc. }
$$

In $\mathbf{P}_{r} \mathbf{P}_{s}-\mathbf{P}_{r-1} \mathbf{P}_{s+1}$ the coefficient of the terms containing $p$ squares will therefore be

$$
\begin{aligned}
& (r+s-2 p) !\{1 /(r-p) !(s-p) !-1 /(r-p-1) !(s-p+1) !\} \\
= & (s-r+1)(r+s-2 p) ! /(r-p) !(s-p+1) !
\end{aligned}
$$

which is positive.

We may put this result in the form

$$
\begin{aligned}
& \mathbf{P}_{r} \mathbf{P}_{s}-\mathbf{P}_{r-1} \mathbf{P}_{s+1} \\
& =\Sigma a_{1}^{2} a_{2}^{2} \ldots a_{r}^{2} a_{r+1} \ldots a_{A} \\
& +(s-r+1) \Sigma a_{1}^{3} a_{2}^{2} \ldots a_{r-1}^{2} a_{r} \ldots a_{s+1}+\ldots \\
& +(s-r+1) \frac{(r+s-2 p) !}{(r-p) !(s-p+1) !} \Sigma a_{1}{ }^{2} a_{2}{ }^{2} \ldots a_{p}{ }^{2} a_{p+1} a_{p+2} \ldots a_{r+a-p}+\ldots .
\end{aligned}
$$

When $r+s \ngtr t$, the last term will be

$$
\frac{(s-r+1)(r+s) !}{r !(s+1) !} \Sigma a_{1} a_{2} \ldots a_{r+s}
$$


but when $r+s>t$, the series will stop when $r+s-p=t$, that is for $p=r+s-t$. If we substitute from (4) in the right of (3) we obtain a formula for the left of (3) as a sum of positive terms. For the case $r=8$ this is Muirhead's formula (33), Vol. XXI., page 156 .

5. From the equation (3) itself we can derive other interesting expansions of $\mathbf{P}_{r} \mathbf{P}_{s}-\mathbf{P}_{r-1} \mathbf{P}_{s+1}$ serving like (4) to show that this function contains only positive terms.

Consider first the form which (3) takes when in addition to the $n$ letters $a, b, c, \ldots$, there are other $v$ letters $a, \beta, \gamma, \ldots$ This may be written

$$
\begin{aligned}
& r(n+v-8) \mathbf{P}_{r} \mathbf{P}_{s}-(n+v-r+1)(s+1) \mathbf{P}_{r-1} \mathbf{P}_{s+1} \\
& =S(a-b)^{2}\left(\mathbf{P}_{r-1}^{a b} P_{a-1}^{a b}-P_{r-2}^{a b} P_{s}^{a b}\right) \\
& +\Sigma(a-a)^{2}\left(\mathbf{P}_{r-1}^{\alpha a} \mathbf{P}_{k-1}^{a a}-\mathbf{P}_{r-2}^{a \alpha} \mathbf{P}_{s}^{a \alpha}\right) \\
& +\Sigma(\alpha-\beta)^{2}\left(P_{r-1}^{\alpha \beta} P_{a-1}^{\alpha \beta}-P_{r-2}^{\alpha \beta} P_{s}^{\alpha \beta}\right) \text {. }
\end{aligned}
$$

Now put $a, \beta, \gamma, \ldots$ all equal to 0 , and this becomes

$$
\begin{aligned}
& r(n+v-s) \mathrm{P}_{r} \mathrm{P}_{s}-(n+v-r+1)(s+1) \mathbf{P}_{r-1} \mathbf{P}_{s+1} \\
= & S(a-b)^{2}\left(\mathbf{P}_{r-1}^{a b} \mathbf{P}_{s-1}^{a b}-\mathbf{P}_{r-2}^{a b} \mathbf{P}_{s}^{a b}\right) \\
+ & v \Sigma a^{2} \quad\left(\mathbf{P}_{r-1}^{a} \mathbf{P}_{s-1}^{a}-\mathbf{P}_{r-2}^{a} \mathbf{P}_{s}^{a}\right)
\end{aligned}
$$

where, of course, only the $n$ letters $a, b, c, \ldots$ are now involved.

$v$ being arbitrary, we must have

$$
r \mathrm{P}_{r} \mathbf{P}_{s}-(s+1) \mathbf{P}_{r-1} \mathbf{P}_{s+1}=\Sigma a^{2}\left(\mathbf{P}_{r-1}^{a} \mathbf{P}_{s-1}^{a}-\mathbf{P}_{r-2}^{a} \mathbf{P}_{s}^{a}\right)
$$

which may be written in either of the forms

$$
\begin{aligned}
& (s+1)\left(\mathrm{P}_{r} \mathrm{P}_{s}-\mathrm{P}_{r-1} \mathrm{P}_{s+1}\right)=(s-r+1) \mathbf{P}_{r} \mathbf{P}_{s}+\sum a^{2}\left(\mathrm{P}_{r-1}^{a} \mathrm{P}_{s-1}^{a}-\mathbf{P}_{r-2}^{a} \mathrm{P}_{a}^{a}\right), \\
& r\left(\mathrm{P}_{r} \mathrm{P}_{2}-\mathrm{P}_{r-1} \mathrm{P}_{a+1}\right)=(s-r+1) \mathrm{P}_{r-1} \mathrm{P}_{s+1}+\Sigma a^{2}\left(\mathrm{P}_{r-1}^{a} \mathrm{P}_{s-1}^{a}-\mathrm{P}_{r-2}^{a} \mathrm{P}_{s}^{a}\right) \text {. }
\end{aligned}
$$

(6) or (6)' may be used as a reduction formula for expressing $P_{r} P_{\imath}-P_{r-1} P_{a+1}$ as a sum of positive terms. Taking (6) we get

$$
\mathbf{P}_{r-1}^{a} \mathbf{P}_{a-1}^{a}-\mathbf{P}_{r-2}^{a} \mathbf{P}_{i}^{a}=\frac{8-r+1}{8} \mathbf{P}_{r-1}^{a} \mathbf{P}_{a-1}^{a}+\frac{1}{8} \sum b^{2}\left(\mathbf{P}_{r-2}^{a b} \mathbf{P}_{-2}^{a b}-\mathbf{P}_{r \rightarrow 3}^{a b} \mathbf{P}_{r-1}^{a b}\right)
$$


and therefore by substitution in (6)

$$
\begin{aligned}
\mathbf{P}_{r} \mathrm{P}_{s}-\mathrm{P}_{r-1} \mathrm{P}_{s+1}=\frac{s-r+1}{s+1} & \mathrm{P}_{r} \mathrm{P}_{s}+\frac{s-r+1}{s . s+1} \Sigma a^{2} \mathrm{P}_{r-1}^{a} \mathrm{P}_{s-1}^{a} \\
& +\frac{2}{s . s+1} \Sigma a^{2} b^{2}\left(\mathbf{P}_{r-2}^{a b} \mathrm{P}_{s-2}^{a b}-\mathrm{P}_{r-3}^{a b} \mathbf{P}_{s-1}^{a b}\right) .
\end{aligned}
$$

We can apply (6) again to the last term, and so on, and obtain finally

$$
\begin{aligned}
& \mathbf{P}_{r} \mathbf{P}_{s}-\mathbf{P}_{r-1} \mathrm{P}_{s+1}=(s-r+1) \frac{1}{s+1} \mathrm{P}_{r} \mathrm{P}_{s}+(s-r+1) \frac{1}{s+1 . s} \mathrm{\Sigma}^{2} \mathbf{P}_{r-1} \mathbf{P}_{s \rightarrow 1}^{a} \\
& +(s-r+1) \frac{1.2}{s+1 . s .8-1} \Sigma a^{2} b^{2} \mathbf{P}_{r-2}^{a b} \mathbf{P}_{a-2}^{a b}+\ldots \ldots \\
& +\frac{r !}{s+1 . s \ldots \ldots(s-r+2)} \Sigma\left(a^{2} b^{2} \ldots, r \text { letters }\right) P_{s-r}^{a b \ldots, r \text { lettern. }}
\end{aligned}
$$

A similar formula can be derived from (6)'.

If we use the formula (7) to develop (3) we get

$$
\begin{aligned}
& \frac{s}{s-r+1}\left\{r(n-s) \mathrm{P}_{r} \mathrm{P}_{s}-(n-r+1)(s+1) \mathrm{P}_{r-1} \mathrm{P}_{s+1}\right\} \\
& =\Sigma(a-b)^{2} \mathbf{P}_{r-1}^{a b} \mathbf{P}_{s-1}^{a b}+\frac{1}{s-1} \mathscr{Y}(a-b)^{2} c^{2} \mathbf{P}_{r-2}^{a b c} \mathbf{P}_{x-2}^{a b c} \\
& +\frac{1.2}{s-1 . s-2} \Sigma(a-b)^{2} c^{2} d^{2} \mathbf{P}_{r-3}^{a b c d} \mathrm{P}_{k-3}^{a b c d}+\ldots \ldots
\end{aligned}
$$

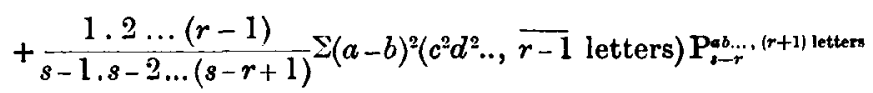

which when $r=s$ becomes Muirhead's (38), page 157, Vol. XXI.

6. By a simple transformation we can derive from (8) another formula which is perhaps simpler and in certain cases will contain fewer terms.

One special case we may note at once. Put $s=n-1$ in (3) and we have inmediately

$$
r \mathrm{P}_{r} \mathrm{P}_{n-1}-(n-r+1) n \mathrm{P}_{r-1} \mathrm{P}_{n}=\Sigma(a-b)^{2} \mathrm{P}_{r-1}^{a b} \mathrm{P}_{n-2}^{a b},
$$

since $\mathrm{P}_{n-1}^{a b}$, referring to only $n-2$ letters, is zero. For this case (8) is clearly a much longer formula.

For the general case, apply (8) not to $a, b, c, \ldots$ themselves, but to their reciprocals $1 / a, 1 / b, 1 / c, \ldots$ 
怤

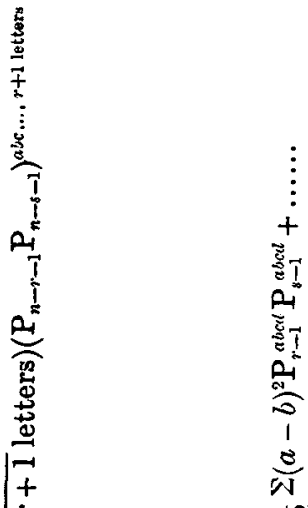

隐

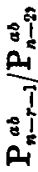
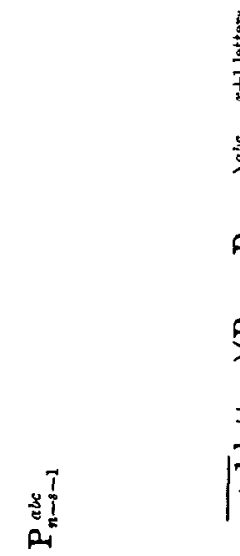

용

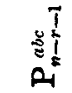

:

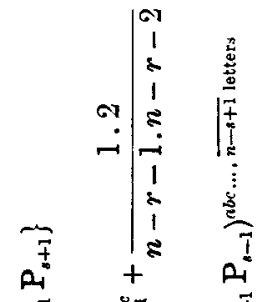

总

$-1 ; 0$

站

है

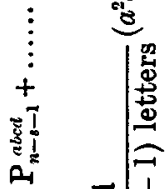

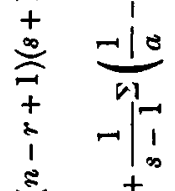

हก

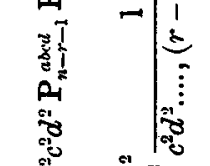

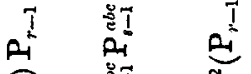

$\sum_{i=1}^{\infty} \quad \frac{1}{1}+$

की

-

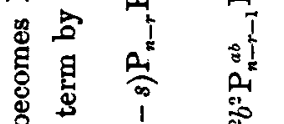

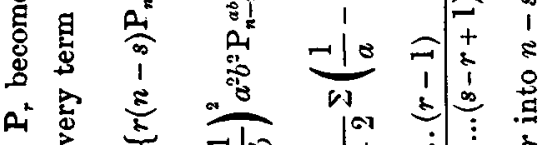

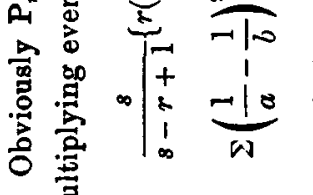

II

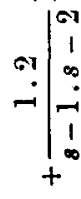

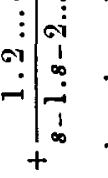

$\because \begin{array}{ll}1 & 3 ! \\ 5 & 81\end{array}$

कo $=\sqrt{1}$ 年

1 1

हो की

$\stackrel{+}{=} \quad \frac{01}{0}$

$\frac{1}{v 1}$

$\begin{array}{llll}2 & + & \text { ह } \\ 8 & i & \text { I }\end{array}$

is

\begin{tabular}{ll|l}
1 & -1 \\
1 & 1 & \\
1 & 0 & 0 \\
1 & 0
\end{tabular}

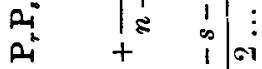

क है। हा

8ा का ह

की 1

$\frac{8}{0}$

1
1
1
+

II

6 Vol. 24 


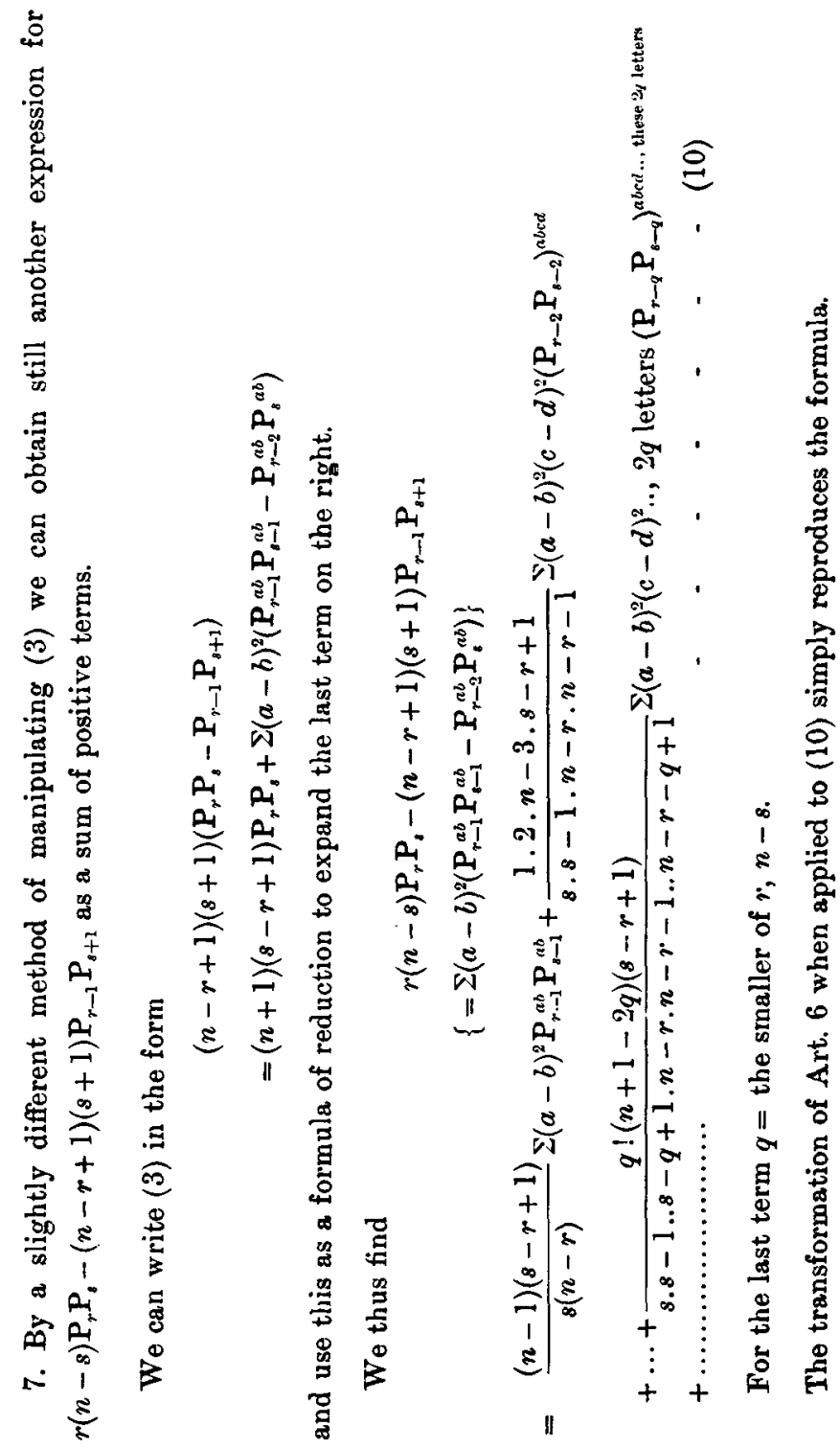


8. The preceding formula, viz., (8), (9), (10) and the result of substituting (4) in (3) furnish various expressions for the difference

$$
r(n-8) \mathrm{P}_{r} \mathrm{P}_{s}-(n-r+1)(s+1) \mathrm{P}_{r-1} \mathrm{P}_{s+1}
$$

as a sum of terms obviously positive when $a, b, c$, etc., are positive and not all equal, and $r$ is less than or equal to $s$.

If as in Art. 2 we put ${ }_{n} \mathrm{C}_{r} . A_{r}$ for $P_{r}$, and so on, this difference takes the form $r(n-8)_{n} \mathrm{C}_{r} \cdot{ }_{n} \mathrm{C}_{z}\left(\mathrm{~A}_{r} \mathrm{~A}_{s}-\mathrm{A}_{r-1} \mathrm{~A}_{s+1}\right)$.

We have thus obtained expressions evidently positive for

$$
\text { and } \begin{gathered}
\mathbf{A}_{r}{ }^{2}-\mathbf{A}_{r-1} \mathbf{A}_{r+1} \\
\mathbf{A}_{r} \mathbf{A}_{s}-\mathbf{A}_{r-1} \mathbf{A}_{s+1} \quad(r<s)
\end{gathered}
$$

thus accounting for cases $(a)$ and $(b)$ of Art. 2.

In accordance with the statement at the end of that Article, the general problem now before us is to throw any difference of the type (e) into the form of a sum of terms such as $\mathbf{K}\left(\mathbf{A}_{r} \mathbf{A}_{s}-\mathbf{A}_{r-1} \mathbf{A}_{s+1}\right)$ where $r \ngtr 8$ and $K$ is a positive product of the powers of the $A^{\prime} s$; a problem of some interest even apart from the application to inequalities.

9. The forms $(c)$ and $(d)$ are particular cases of $(e)$, but as they are specially simple and interesting, we shall deal with them here individually before proceeding to a method applicable to the general form.

We have immediately

$$
\begin{aligned}
& A_{r} A_{d}-A_{r-p} A_{d+p}=\left(A_{r} A_{d}-A_{r-1} A_{s+1}\right)+\left(A_{r-1} A_{s+1}-A_{r-2} A_{s+2}\right)+\ldots \\
& +\left(A_{r-p+1} A_{s+p-1}-A_{r-p} A_{s+p}\right) \text {. } \\
& \mathbf{A}_{r}^{r+1}-\mathbf{A}_{0} \mathbf{A}_{r+1}^{r}=\left(\mathbf{A}_{r}^{r+1}-\mathbf{A}_{r-1} \mathbf{A}_{r}^{r-1} \mathbf{A}_{r+1}\right)+\left(\mathbf{A}_{r-1} \mathbf{A}_{r}^{r-1} \mathbf{A}_{r+1}-\mathbf{A}_{r-2} \mathbf{A}_{r}{ }^{r-2} \mathbf{A}_{r+1}^{2}\right) \\
& +\left(A_{r-2} A_{r}^{r-2} A_{r+1}^{2}-A_{r-3} A_{r}^{r-3} A_{r+1}^{3}\right)+\ldots \ldots \\
& +\left(A_{2} A_{r}{ }^{2} A_{r+1}^{r-2}-A_{1} A_{r} A_{r+1}^{r-1}\right)+\left(A_{1} A_{r} A_{r+1}^{r-1}-A_{0} A_{r+1}^{r}\right) \\
& =A_{r}^{r-1}\left(A_{r}^{2}-A_{r-1} A_{r+1}\right)+A_{r}^{r-2} A_{r+1}\left(A_{r-1} A_{r}-A_{r-2} A_{r+1}\right) \\
& +A_{r}^{r-3} A_{r+1}^{2}\left(A_{r-2} A_{r}-A_{r-3} A_{r+1}\right)+\ldots \ldots \\
& +A_{r} A_{r+1}^{r-2}\left(A_{2} A_{r}-A_{1} A_{r+1}\right)+A_{r+1}^{r-1}\left(A_{1} A_{r}-A_{0} A_{r+1}\right) \text {. - }
\end{aligned}
$$


10. The general difference is what is obtained from

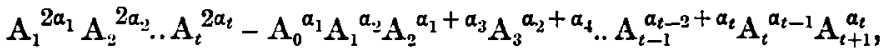
when all factors, in the form of powers of $A$ 's, which are common to the two terms are removed.

Some of the a's may not occur, that is, they nay be zero; but none of them are to be negative; when this condition is fulfilled we shall call (13) or (13) simplified a proper difference.

Even after simplification, note that if $a_{p}$ and $a_{t}$ are the $a^{\prime} s$ of Jowest and highest order, then $A_{p-1}$ and $A_{t+1}$ are the lowest and highest A's which occur, and they occur in the negative term in the form $A_{p-1}^{\alpha_{p}}, A_{t+1}^{\alpha_{t}}$.

Any difference being given, even in its simplified form, its a's are determinate and can easily be found. Thus suppose that a given difference is

$$
\mathbf{A}_{1}^{x_{1}} \mathbf{A}_{2}^{x_{2}} \ldots \mathbf{A}_{t}^{x_{t}}-\mathbf{A}_{0}{ }^{y_{0}} \mathbf{A}_{1}{ }_{1}^{y_{1}} \mathbf{A}_{2} y_{2} \ldots \mathbf{A}_{t}^{y_{t}} \mathbf{A}_{t+1}^{y_{t+1}}
$$

where, if the difference has been simplified, one of $x_{r}, y_{r}$ is zero.

If this is derivable from (13) by mere elision of common factors, then

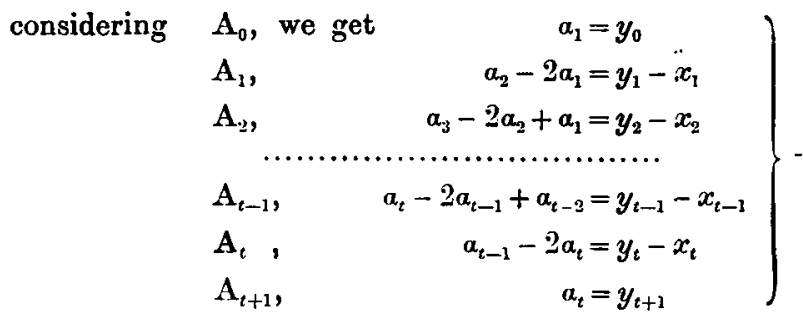

The first $t$ of these equations give in succession $\alpha_{1}, a_{2}, \ldots, a_{t}$. The remaining two equations give

$$
y_{0}+\left(y_{1}-x_{1}\right)+\left(y_{2}-x_{i}\right)+\ldots+\left(y_{t}-x_{t}\right)+y_{t+1}=0
$$

and $\quad\left(y_{1}-x_{1}\right)+2\left(y_{2}-x_{2}\right)+3\left(y_{3}-x_{3}\right)+\ldots+t\left(y_{t}-x_{t}\right)+(t+1) y_{t+1}=0$. These are the conditions that (14) should be derivable from a form of the type (13), and express the facts that the two terms of (14) are homogeneous in the $\mathrm{A}^{\prime} \mathrm{s}$ and also in $a, b, c, \ldots$ 
The values of the a's are easily found explicitly from (15). Thus

$$
\begin{aligned}
a_{1} & =y_{0}, \\
a_{3}-a_{1} & =y_{0}+\left(y_{1}-x_{1}\right), \\
a_{3}-a_{2} & =y_{0}+\left(y_{1}-x_{1}\right)+\left(y_{2}-x_{2}\right), \text { etc. }
\end{aligned}
$$

so that $a_{1}=y_{0}$,

$$
\left.\begin{array}{l}
a_{2}=2 y_{0}+\left(y_{1}-x_{1}\right), \\
a_{3}=3 y_{0}+2\left(y_{1}-x_{1}\right)+\left(y_{2}-x_{2}\right), \\
a_{4}=4 y_{0}+3\left(y_{1}-x_{1}\right)+2\left(y_{2}-x_{2}\right)+\left(y_{3}-x_{3}\right), \text { etc. }
\end{array}\right\}
$$

For example, for $\quad A_{r} A_{s}-A_{r-p} A_{s+\mu} \quad(s \nless r)$ the $a$ 's that occur are

$$
\left.\begin{array}{l}
a_{r-p+1}=1, \alpha_{r-p+2}=2, a_{r-p+3}=3, \ldots, a_{r}=p ; \\
a_{r+1}=a_{r+2}=\ldots=a_{s}=p ; \\
a_{s+1}=p-1, a_{s+2}=p-2, \ldots, a_{s+p-1}=1 .
\end{array}\right\}
$$

In particular, for

$$
\mathbf{A}_{r} \mathbf{A}_{\mathbf{s}}-\mathbf{A}_{r-1} \mathbf{A}_{s+1}
$$

$$
a_{r}=\alpha_{r+1}=a_{r+2}=\ldots=\alpha_{s}=1 \text {. }
$$

Again for

$$
\mathbf{A}_{r}^{r+1}-\mathbf{A}_{0} \mathbf{A}_{r+1}^{r}
$$

$$
a_{1}=1, a_{2}=2, a_{3}=3, \ldots, a_{r}=r \text {. }
$$

11. It is now easy to explain a method of reducing any given proper difference (14) to the shape we desire.

For brevity, denote the given difference by $\mathrm{X}-\mathrm{Y}$.

Then the process we will give is a step-by-step one, the first step being to put $X-Y$ in the form

$$
\left(\mathrm{X}-\mathrm{Y}_{1}\right)+\left(\mathrm{Y}_{1}-\mathrm{Y}\right)
$$

where (i) $Y_{1}-Y$ is the product of powers of $A$ 's by a proper difference of the elementary type $A_{r} A_{1}-A_{r-1} A_{s+1}$

and (ii) $X-Y_{1}$ is a proper difference with some of its a's smaller, and none larger, than those of $X-Y$.

Suppose that $A_{r-1} A_{c+1}$ occurs in $Y$ as a factor.

For $Y_{1}$ take what $Y$ becomes when this factor is replaced by A,A, 
Then (i) $Y_{1}-\mathrm{Y}$ is clearly $\left(\mathbf{A}_{r} \mathbf{A}_{s}-\mathbf{A}_{r-1} A_{s+1}\right) \times$ a product of powers of $A^{\prime}$ 's,

and (ii) $\mathrm{X}-\mathrm{Y}_{1}$ is a difference in which the values of all the a's from $\alpha_{r}$ to $\alpha_{z}$ inclusire are reduced by 1 from their values in $\mathrm{X}-\mathrm{Y}$, the other a's remaining unchanged; as may easily be verified from equations (16).

More generally, if $\left(\mathrm{A}_{r-1} \mathbf{A}_{s+1}\right)^{m}$ occurs in $\mathbf{Y}$ as a factor, and we form $Y_{1}$ by replacing this by $\left(A_{r} A_{x}\right)^{m}$, then

(i) $\mathbf{Y}_{1}-\mathbf{Y}$ will be $\left(\mathbf{A}_{r}{ }^{m} \mathbf{A}_{x}{ }^{m}-\mathbf{A}_{r-1}^{m} \mathbf{A}_{s+1}\right) \times$ a product of powers of $A^{\prime}$ 's, that is, $\left(A_{r} A_{s}-A_{r-1} A_{s+1}\right) \times$ an integral function of $A$ 's with positive coefficients ;

and (ii) $\mathrm{X}-\mathrm{Y}_{1}$ will have all the $a$ 's from $\alpha_{r}$ to $a_{s}$ inclusive reduced by $m$ from their values in $\mathrm{X}-\mathrm{Y}$.

For the success of this process of reduction based on replacing $A_{r-1} A_{b+1}$ in $Y$ by $A_{r} A_{a}$ it is necessary

(i) that $A_{r-1} A_{s+1}$ should occur in $\mathrm{Y}$;

(ii) that $\mathrm{X}-\mathrm{Y}_{1}$ should still be a proper difference, which requires that none of the new $a$ 's should be negative, or that in $\mathrm{X}-\mathrm{Y}$ all the $\alpha^{\prime} \mathrm{s}$ from $a_{r}$ to $a_{a}$ inclusive should occur.

Both these conditions will be fulfilled if we take for $r$ the index of the lowest $\alpha$ that occurs in $\mathbf{X}-\mathbf{Y}$, in which case $\mathbf{A}_{r-1}$ certainly occurs in $Y$, as pointed out near the beginning of Art. 10; and if at the same time we choose $s$ so that $a_{t+1}$ is the first $a$ after $a_{r}$ which does not occur in $Y$, in which case $A_{t+1}$ certainly occurs in $Y$, viz., raised to the power $a_{s}+a_{s+2}$, as we see from (13) since $A_{t+1}$ does not occur in $\mathrm{X}$.

More generally, we can replace at one step $\left(A_{r-1} A_{t+1}\right)^{m}$ by $\left(\mathbf{A}_{r} \mathbf{A}_{t}\right)^{m}$ provided, with $r$ and $s$ chosen as just explained, every $a$ from $\alpha_{r}$ to $\alpha_{\text {a }}$ inclusive is equal to $m$ at least.

Having thus got $X-Y=\left(X-Y_{1}\right)+\left(Y_{1}-Y\right)$ we proceed in the same way with $X-Y_{1}$ and finally obtain

$$
\mathrm{X}-\mathrm{Y}=\left(\mathrm{X}-\mathrm{Y}_{n}\right)+\left(\mathrm{Y}_{n}-\mathrm{Y}_{n-1}\right)+\ldots+\left(\mathrm{Y}_{2}-\mathrm{Y}_{1}\right)+\left(\mathrm{Y}_{1}-\mathrm{Y}\right)
$$


where each of the differences in brackets is of the type

$$
\left(A_{r} A_{s}-A_{r-1} A_{s+1}\right) \times
$$

an integral function of $A$ 's with positive coefficients.

The reader may take the results of Art. 9 as easy examples of this process.

As another example consider the case

$$
X=A_{2}{ }^{7} A_{4}{ }^{4} A_{8}{ }^{4} ; \quad Y=A_{0} A_{1}{ }^{4} A_{3}{ }^{3} A_{5}{ }^{4} A_{7}{ }^{3} \text {. }
$$

The scheme of $a$ 's, originally, and after each step, is

\begin{tabular}{ll|l|l|l|l|l} 
& $a_{1}$ & $a_{2}$ & $a_{3}$ & $a_{4}$ & $a_{5}$ & $a_{6}$ \\
$\mathrm{X}-\mathrm{Y}$. & 1 & 6 & 4 & 5 & 2 & 3 \\
$\mathrm{X}-\mathrm{Y}_{1}$. & & 5 & 3 & 4 & 1 & 2 \\
$\mathrm{X}-\mathrm{Y}_{2}$. & & 4 & 2 & 3 & & 1 \\
$\mathrm{X}-\mathrm{Y}_{3}$. & & 2 & & 1 & & 1 \\
$\mathrm{X}-\mathrm{Y}_{4}$. & & & & 1 & & 1 \\
$\mathrm{X}-\mathrm{Y}_{5}$. & & & & & & 1
\end{tabular}

Therefore

and

$$
\begin{aligned}
& Y_{1}=A_{1}{ }^{5} A_{3}{ }^{3} A_{5}{ }^{4} A_{6} A_{7}{ }^{2} \\
& Y_{2}=A_{1}{ }^{4} A_{2} A_{3}{ }^{3} A_{5}{ }^{4} A_{6}{ }^{2} A_{i} \\
& Y_{3}=A_{1}{ }^{2} A_{2}{ }^{3} A_{3}{ }^{3} A_{4}{ }^{2} A_{5}{ }^{2} A_{6}{ }^{2} A_{7} \\
& Y_{4}=A_{2}{ }^{7} A_{3} A_{4}{ }^{2} A_{5}{ }^{2} A_{6}{ }^{2} A_{7} \\
& Y_{5}=A_{2}{ }^{7} A_{4}{ }^{4} A_{5} A_{6}{ }^{2} A_{7}
\end{aligned}
$$

$$
\begin{aligned}
& \mathbf{X}-\mathbf{Y}=\left(\mathbf{X}-\mathbf{Y}_{5}\right)+\left(\mathrm{Y}_{5}-\mathbf{Y}_{4}\right)+\left(\mathrm{Y}_{4}-\mathrm{Y}_{3}\right)+\left(\mathrm{Y}_{3}-\mathbf{Y}_{2}\right)+\left(\mathrm{Y}_{2}-\mathbf{Y}_{1}\right)+\left(\mathrm{Y}_{1}-\mathbf{Y}\right) \\
& =A_{2}{ }^{7} A_{4}{ }^{4} A_{6}{ }^{2}\left(A_{6}{ }^{2}-A_{5} A_{7}\right) \\
& +A_{2}{ }^{7} A_{4}{ }^{2} A_{5} A_{6}{ }^{2} A_{7}\left(A_{4}{ }^{2}-A_{3} A_{6}\right) \\
& +A_{2}{ }^{3} A_{3} A_{4}{ }^{2} A_{5}{ }^{2} A_{6}{ }^{2} A_{7}\left(A_{3}{ }^{4}-A_{1}{ }^{2} A_{3}{ }^{2}\right) \\
& +A_{1}{ }^{2} A_{2} A_{3}{ }^{3} A_{5}{ }^{3} A_{6}{ }^{2} A_{7}\left(A_{2}{ }^{2} A_{4}{ }^{2}-A_{1}{ }^{2} A_{5}{ }^{2}\right) \\
& +A_{1}{ }^{4} A_{3}{ }^{3} A_{5}{ }^{4} A_{6} A_{7}\left(A_{2} A_{6}-A_{1} A_{7}\right) \\
& +A_{1}{ }^{4} A_{3}{ }^{3} A_{5}{ }^{4} A_{7}{ }^{2}\left(A_{1} A_{6}-A_{0} A_{7}\right) \text {, }
\end{aligned}
$$

where we may replace

$$
A_{2}{ }^{4}-A_{1}{ }^{2} A_{3}{ }^{2} \text { by }\left(A_{2}{ }^{2}+A_{1} A_{3}\right)\left(A_{2}{ }^{2}-A_{1} A_{3}\right)
$$

and

$$
A_{2}{ }^{2} A_{4}{ }^{2}-A_{1}{ }^{2} A_{5}{ }^{2} \text { by }\left(A_{9} A_{4}+A_{1} A_{5}\right)\left(A_{2} A_{4}-A_{1} A_{5}\right) \text {. }
$$


12. The preceding process for decomposing a "proper difference" is simple and straightforward, but it is not to be inferred that the decomposition obtained is the only one possible which would serve the purpose. Take for example

As in Art. 9 this is

$$
\mathbf{A}_{3}{ }^{4}-\mathbf{A}_{0} \mathbf{A}_{6}{ }^{3} \text {. }
$$

$$
A_{3}{ }^{2}\left(A_{3}{ }^{2}-A_{2} A_{4}\right)+A_{3} A_{4}\left(A_{2} A_{3}-A_{1} A_{4}\right)+A_{4}{ }^{2}\left(A_{1} A_{3}-A_{0} A_{4}\right) .
$$

But it may also be put in the form

$$
\left(A_{3}{ }^{2}+A_{2} A_{4}\right)\left(A_{3}{ }^{2}-A_{2} A_{4}\right)+A_{4}{ }^{2}\left(A_{2}{ }^{2}-A_{1} A_{3}\right)+A_{4}{ }^{2}\left(A_{1} A_{3}-A_{0} A_{4}\right) \text {. }
$$

The latter form, however, contains a term more than the other, if we count $\left(A_{r} A_{t}-A_{r-1} A_{c+1}\right)$ as one term, but $\left(A_{r}{ }^{m} A_{s}{ }^{m}-A_{r-1}{ }^{m} A_{s+1}\right)$ ), i.e., $\left(A_{r} A_{s}-A_{r-1} A_{a+1}\right)\left(A_{r}^{m-1} A_{c}^{m-1}+\ldots\right)$ as $m$ terms.

So, generally, it may happen that it is possible to make two or more steps of the single step by which in the above process we change $Y_{a}$ into $Y_{b}$ by replacing $A_{r-1} A_{s+1}$ by $A_{r} A_{d}$. For if an $A$, say $A_{x+1}$, intermediate to $A_{r-1}$ and $A_{s+1}$ occur in $Y_{a}$, as it may very well do, we may first change $Y_{a}$ into $Y_{c}$, say, by replacing $A_{r-1} A_{x+1}$ by $A_{r} A_{x}$, and then change $Y_{c}$ into $Y_{b}$ by replacing $A_{x} A_{a+1}$ by $A_{x+1} A_{a}$. The only effect of this is to split up a single term of the original final result into two, and therefore to increase the total number of terms by one.

It would therefore seem that the decomposition we have given is that which leads to the minimum number of terms.

13. An inequality of perennial interest is that which holds between the Arithmetic and Geometric Means of a set of positive numbers, and we may conclude by examining what the preceding methods make of this important example.

The Arithmetic Mean of the $n$ numbers $a, b, c, \ldots$ is

$$
(a+b+c+\ldots) / n \text { or } \mathbf{A}_{1}
$$

their Geometric Mean is $(a b c \ldots)^{1 / n}$, i.e., $\mathbf{A}_{n}^{1 / n}$.

We prove $A_{1}>A_{n}{ }^{1 / n}$ by exhibiting $A_{1}{ }^{n}-A_{n}$ in an explicitly positive form. 
To look for a moment at a more general case, note that by (d)

\begin{tabular}{|c|c|c|}
\hline and therefore & $\begin{array}{l}A_{r}^{1 / r}>A_{r+1}^{1 / \sqrt{r+1}} \\
A^{1 / r}>A^{1 / r} \text { if } 8>r:\end{array}$ & \\
\hline hence & $\mathbf{A}_{r}^{s}-\mathbf{A}_{s}^{r}$ or $\mathbf{A}_{r}^{+}-\mathbf{A}_{0}^{r-r} \mathbf{A}_{s}^{r}$ is positive, & \\
\hline is, in fact, a $p r$ & oper difference when $s>r$ & \\
\hline The $a$ 's of $t$ & bis difference are by $(16)$ & \\
\hline$a_{1}=8-r$ & $a_{2}=2(s-r), a_{3}=3(s-r), \ldots, a_{r}$ & $=r(s-r)$; \\
\hline
\end{tabular}

If $r$ and $s$ are given numerically, the reduction by the method of Art. 11 is very simple ; for, since the $a^{\prime}$ 's diminish steadily from $a_{r}$ towards both ends of the series, it is clear that at every step we have merely to replace in the negative term the lowest $A$ by the $A$ one higher and the highest $\mathrm{A}$ by the $\mathrm{A}$ one lower. But it would be somewhat difficult to state a general formula for the result, the form of which in fact depends on the relative magnitudes of the various multiples of $r$ and $s$.

For the special case $\mathbf{A}_{1}{ }^{n}-\mathbf{A}_{0}{ }^{n-1} \mathbf{A}_{n}$ the difficulty does not exist.

The a's are $a_{1}=n-1, a_{2}=n-2, a_{3}=n-3, \ldots, a_{n}=1$;

and the formula, which may be rerified at a glance without reference to the general method, is

$$
\begin{aligned}
A_{1}{ }^{n}-A_{n}= & A_{1}{ }^{n-2}\left(A_{1}{ }^{2}-A_{2}\right)+A_{1}{ }^{n-3}\left(A_{1} A_{2}-A_{3}\right)+A_{1}{ }^{n-4}\left(A_{1} A_{3}-A_{4}\right)+\ldots \\
& +A_{1}\left(A_{1} A_{n-2}-A_{n-1}\right)+\left(A_{1} A_{n-1}-A_{n}\right) .
\end{aligned}
$$

Now (3) of Art. 3 gives

$$
\begin{gathered}
(n-8) \mathrm{P}_{1} \mathrm{P}_{s}-n(s+1) \mathrm{P}_{a+1}=\Sigma(a-b)^{2} \mathrm{P}_{s-1}^{a b} \\
\text { i.e., } \quad n^{2} \frac{(n-1)(n-2) \ldots(n-8)}{1.2 \ldots \ldots 8}\left(\mathrm{~A}_{1} \mathrm{~A}_{s}-\mathrm{A}_{s+1}\right)=\Sigma(a-b)^{2} \mathrm{P}_{s \rightarrow 1}^{a b} .
\end{gathered}
$$

Hence

$$
\begin{aligned}
A_{1}{ }^{n}-A_{n}=\frac{1}{n^{2}} \Sigma(a-b)^{2}\left\{\frac{1}{n-1} A_{1}{ }^{n-2}\right. & +\frac{1.2}{n-1 \cdot n-2} \quad A_{1}{ }^{n-3} P_{1}^{a b} \\
& \left.+\frac{1.2 \cdot 3}{n-1 \cdot n-2 \cdot n-3} A_{1}^{n-4}{P_{2}{ }^{a b}+\ldots}^{a b}+\frac{1}{n-1} A_{1} P_{n-3}^{a b}+P_{n-2}^{a b}\right\}
\end{aligned}
$$

